\title{
Technologies optiques, CD-ROM et bibliothèques
}

Partie 2. Enquête sur l'utilisation du CD-ROM dans les

bibliothèques du Québec

Optical Technologies, CD-ROM and Libraries

Part 2. Survey of CD-ROM Use in Québec Libraries

Tecnologías ópticas, CD-ROM y bibliotecas

Parte 2. Encuesta sobre la utilización del CD-ROM en las

bibliotecas quebequenses

\section{Gilles Deschatelets}

Volume 35, numéro 3, juillet-septembre 1989

URI : https://id.erudit.org/iderudit/1028148ar

DOI : https://doi.org/10.7202/1028148ar

Aller au sommaire du numéro

\section{Éditeur(s)}

Association pour l'avancement des sciences et des techniques de la documentation (ASTED)

\section{ISSN}

0315-2340 (imprimé)

2291-8949 (numérique)

Découvrir la revue

\section{Citer cet article}

Deschatelets, G. (1989). Technologies optiques, CD-ROM et bibliothèques : partie 2. Enquête sur l'utilisation du CD-ROM dans les bibliothèques du Québec.

Documentation et bibliothèques, 35(3), 79-96. https://doi.org/10.7202/1028148ar
Résumé de l'article

Ce second article présente les résultats d'une enquête auprès de toutes les bibliothèques et centres de documentation du Québec sur l'utilisation de produits documentaires sur CD-ROM. Après avoir établi l'importance du CD-ROM comme produit documentaire et donné une vue d'ensemble de son implantation aux États-Unis et au Canada, l'auteur présente les caractéristiques du marché du CD-ROM dans les bibliothèques et passe en revue les différentes enquêtes réalisées à ce jour. Le marché québécois du CD-ROM est présenté comme un marché encore marginal et en pleine implantation. Seulement 7,7\% des bibliothèques du Québec offrent un service de CD-ROM, la plupart étant des bibliothèques universitaires ou spécialisées. L'implantation d'un service de CD-ROM est présentée comme étant très étroitement reliée à l'implantation d'un service de téléréférence.
Tous droits réservés (c) Association pour l'avancement des sciences et des techniques de la documentation (ASTED), 1989
Ce document est protégé par la loi sur le droit d'auteur. L'utilisation des services d'Érudit (y compris la reproduction) est assujettie à sa politique d'utilisation que vous pouvez consulter en ligne.

https://apropos.erudit.org/fr/usagers/politique-dutilisation/ 


\title{
Technologies optiques, CD-ROM et bibliothèques.
}

\author{
Partie 2 : Enquête sur I'utilisation du CD-ROM \\ dans les bibliothèques du Québec
}

\author{
Gilles Deschatelets* \\ École de bibliothéconomie et des sciences de l'information \\ Université de Montréal
}

\begin{abstract}
Ce second article présente les résultats d'une enquête auprès de toutes les bibliothèques et centres de documentation du Québec sur l'utilisation de produits documentaires sur CD-ROM. Après avoir établi l'importance du CD-ROM comme produit documentaire et donné une vue d'ensemble de son implantation aux États-Unis et au Canada, l'auteur présente les caractéristiques du marché du CD-ROM dans les bibliothèques et passe en revue les différentes enquêtes réalisées à ce jour. Le marché québécois du $C D-R O M$ est présenté comme un marché encore marginal et en pleine implantation. Seulement $7,7 \%$ des bibliothèques du Québec offrent un service de $C D-R O M$, la plupart étant des bibliothèques universitaires ou spécialisées. L'implantation d'un service de CD-ROM est présentée comme étant très étroitement reliée à l'implantation d'un service de téléréférence.
\end{abstract}

Optical Technologies, CD-ROM and Libraries. Part 2: Survey of CD-ROM Use in Québec Libraries.

This second article summarizes the results of a mail survey of all libraries and documention centres in Québec on the use of CD-ROMs. The author first establishes the importance of CD-ROM as an information resource and presents an overview of its use in American and Canadian libraries. He pinpoints the CD-ROM market's most important characteristics and summarizes the results of various surveys on CD-ROMs. The Québec CDROM market is presented as a small one still in its implementation phase. Only 7,7\% of libraries surveyed have $C D-R O M s$; most of them academic or special libraries. The implementation of CD-ROM services in libraries is compared to that of on-line services.
Tecnologías ópticas, CD-ROM y bibliotecas. Parte 2: Encuesta sobre la utilización del CD. ROM en las bibliotecas quebequenses.

Este segundo artículo presenta los resultados de una encuesta efectuada en todas las bibliotecas y centros de documentación de la provincia de Quebec en cuanto a la utilización de productos documentales sobre CD-ROM. En primer lugar, el autor insiste sobre la importancia del CD-ROM como producto documental y da una visión global de su implantación en los Estados Unidos y el Canadá. A continuación, presenta las características del mercado del CD-ROM en las bibliotecas y resume las distintas encuestas efectuadas hasta hoy. El mercado quebequense del CD-ROM es actualmente un mercado aun marginal $y$ en plena implantación. Solamente un $7,7 \%$ de las bibliotecas quebequenses ofrece el servicio $C D$ ROM y la majoria de ellas son bibliotecas universitarias o especializadas. La implantación de un servicio CD-ROM se liga muy estrechamente a la implantación de un servicio de telereferencia.
Dans un premier article 1 , nous avons retracé la chronologie des disques optiques et nous en avons établi une typologie ${ }^{2}$; nous avons également défini les principales caractéristiques techniques, les avantages et les inconvénients des CD-ROM; et enfin, nous avons présenté les grandes lignes du marché des CD-ROM et de ses applications possibles pour les bibliothèques.

Le présent article discutera les résultats d'une enquête réalisée en avril 1989 auprès de toutes les bibliothèques et centres de documentation du

* L'auteur est professeur agrégé à l'École de bibliothéconomie et des sciences de l'information à l'Université de Montréal.

1. Gilles Deschatelets et Marcel Simoneau, «Technologies optiques, CD-ROM et bibliothèques. Partie 1 : Caractéristiques, marché et applications ", Documentation et bibliothèques, vol. 34, no 2 (avril-juin 1988), 43-71.

2. Voir également l'excellent article de Susan Motley, «CDROM : 1988 in Review ", Computers in Libraries, vol. 9, no. 7 (July-August 1988), 17-19. 
Québec, sur l'utilisation de cette nouvelle technologie. Nous essaierons également de comparer le marché québécois au marché canadien et américain et nous tenterons de dresser un scénario d'implantation probable du CD-ROM dans les bibliothèques.

Dans un troisième article, nous aborderons la question de l'évaluation des produits sur CD-ROM et nous en proposerons une grille d'évaluation.

\section{Importance des CD-ROM}

L'un des principaux indicateurs de la percée d'une nouvelle technologie ou d'un nouveau produit dans un marché est le nombre de documents publiés dans les principales revues constituant son domaine d'application. Ainsi, depuis 1986, il existe déjà une littérature fort imposante sur les CD-ROM. Une recherche automatisée dans 13 bases de données offertes par le serveur Dialog 3 montrait, en avril 1989, un total de 8391 articles ou documents publiés sur les disques et technologies optiques entre 1986 et 1988 (inclusivement). II faut noter

\section{Figure 1}

Bases de données sur CD-ROM

\begin{tabular}{|c|c|c|c|c|c|}
\hline Sources & \multicolumn{5}{|c|}{$\begin{array}{l}\text { Nombre de bases de données } \\
1985 \quad 1986 \quad 1987 \quad 19881989\end{array}$} \\
\hline $\begin{array}{l}\text { Optical } \\
\text { Publishing } \\
\text { Directory }\end{array}$ & 1 & 42 & 84 & 196 & 320 \\
\hline $\begin{array}{l}\text { CD-ROMs in } \\
\text { Print }^{5}\end{array}$ & & & 120 & 175 & 235 \\
\hline $\begin{array}{l}\text { CD-ROM } \\
\text { Databases }^{6}\end{array}$ & & & & & 265 \\
\hline $\begin{array}{l}\text { CD-ROM } \\
\text { Directory }\end{array}$ & & & & 189 & 390 \\
\hline
\end{tabular}

toutefois qu'il existe une certaine redondance quant au nombre d'articles entre les bases de données consultées. Sur les CD-ROM seulement, 3323 documents ont été recensés pour cette période. Le phénomène est donc important et il est permis de croire qu'il n'en est encore qu'à ses débuts.

Timorés ou peut-être tout simplement prudents au départ, les producteurs de bases de données ont laissé tomber leurs réserves initiales pour se lancer dans I' «aventure » du CD-ROM. Déjà selon certaines sources (fig. 1), on recense près de 400 bases de données commerciales sur CD-ROM et le chiffre croît de façon constante.

On peut distribuer ces bases de données selon leur contenu (fig.2), les disciplines couvertes (fig.3), leur fréquence de mise à jour (fig.4) et le nombre de disques utilisés par base de données (fig.5). Ainsi, on constate qu'à l'heure actuelle, $67 \%$ des bases de données sur CD-ROM sont soit bibliographiques, soit des ouvrages de référence, qu'elles couvrent bien davantage le secteur scientifique et les généralités (surtout pour les ouvrages de référence), qu'elles sont, pour la plupart, mises à jour trimestriellement $(48 \%)$ ou annuellement (18\%), donc assez peu fréquemment, et que, dans une proportion de $84 \%$, elles sont offertes sur un seul disque.

Le parallèle est assez saisissant avec les débuts de la recherche en ligne où les premières bases de données étaient surtout bibliographiques et couvraient presque exclusivement le domaine scientifique. La comparaison est d'autant plus pertinente que près de $100 \%$ des contenus des bases de données sur CD-ROM proviennent soit de sources imprimées, soit de sources ordinolingues, soit des deux à la fois ${ }^{8}$. À la différence de la téléréférence, toutefois, les CD-ROM sont faits sur mesure pour les ouvrages de référence où le temps de consultation ne doit pas être un facteur économique discriminant et où la fréquence des mises à jour n'a pas à être trop élevée.
3. Eric, Lisa Isa, Inspec, Abi/Inform, Computer Database, Pascal, Microcomputer Index, Dissertation Abstracts, Current Technology, NTIS, Conference Papers Index et Books in Print.

4. R.A. Bowers, ed., Optical Publishing Directory 1988 , Medford, N.J., Learned Information, 1988.

5. Jean-Paul Emard, ed., CD-ROMS in Print 1988-1989: An International Guide, Westport, CT, Meckler, 1988.

6. $C D-R O M$ Databases, base de données mensuelle publiée par Worldwide Videotex, Boston, MA (sur NewsNet, en date du 10 avril 1989).

7. Erin Cormach, ed., CD-ROM Directory 1989, London, TFPL Publishing, 1988.
8. Paul Travis Nicholls, "Statistical Profile of Currently Available CD-ROM Database Products", Laserdisk Professional, vol. 1 no. 4 (November 1988), 38-45. 
Figure 2

Distribution des bases de données sur CD-ROM par catégories

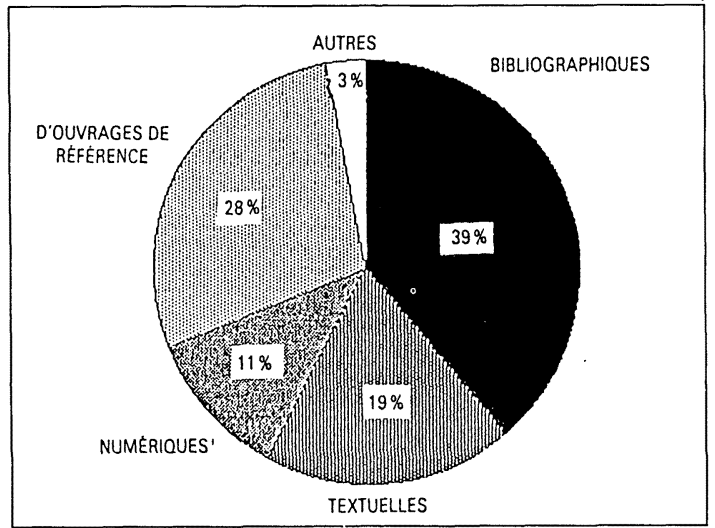

Figure 3

Distribution des bases de données sur CD-ROM par discipline

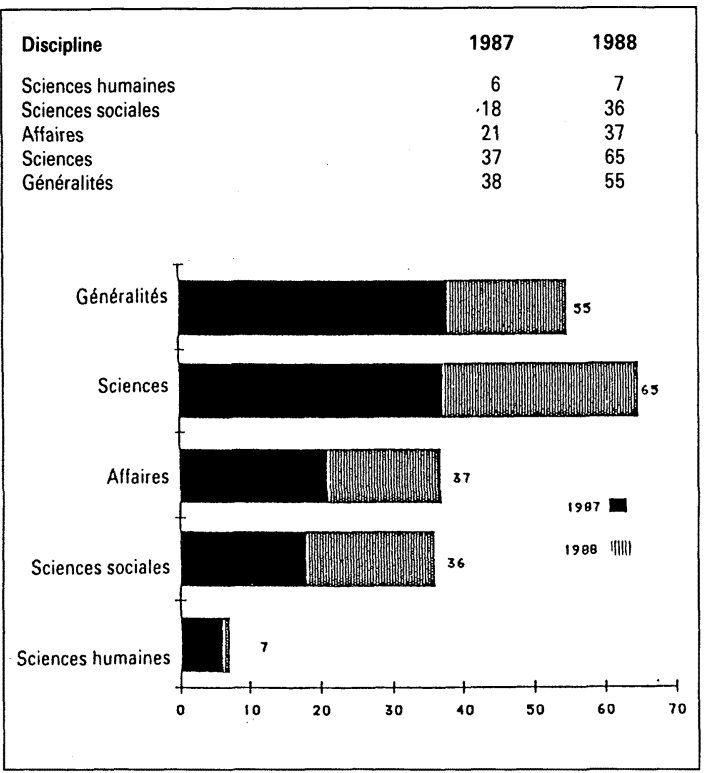

Figure 4

Bases de données sur CD-ROM : fréquence des mises à jour

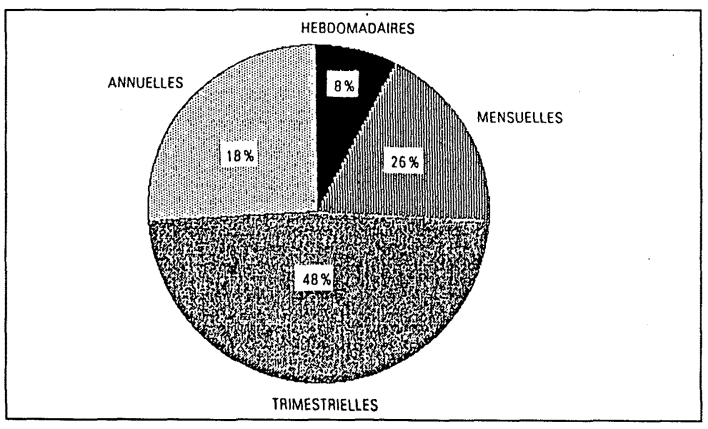

Figure 5

Nombre de disques par base de données sur CD-ROM

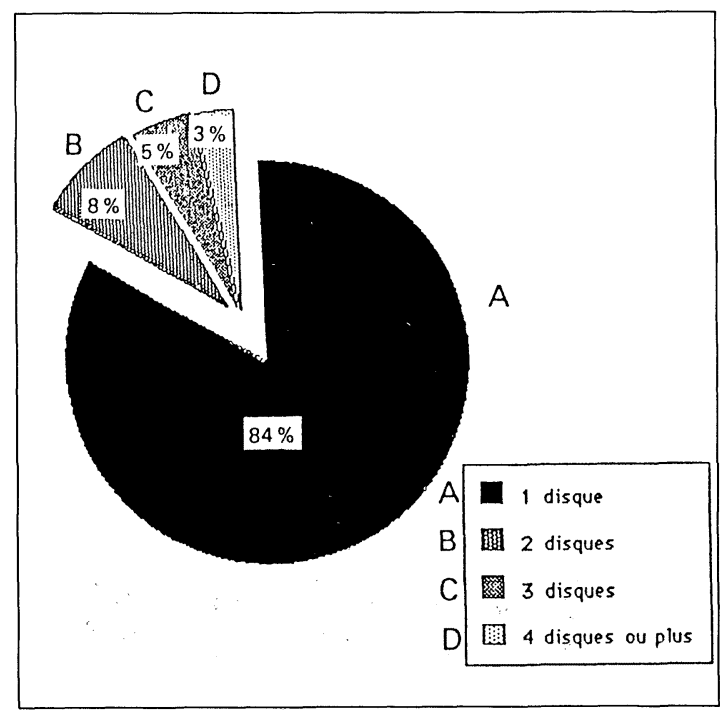

Figure 6

Bases de données sur CD-ROM : coût moyen par catégorie

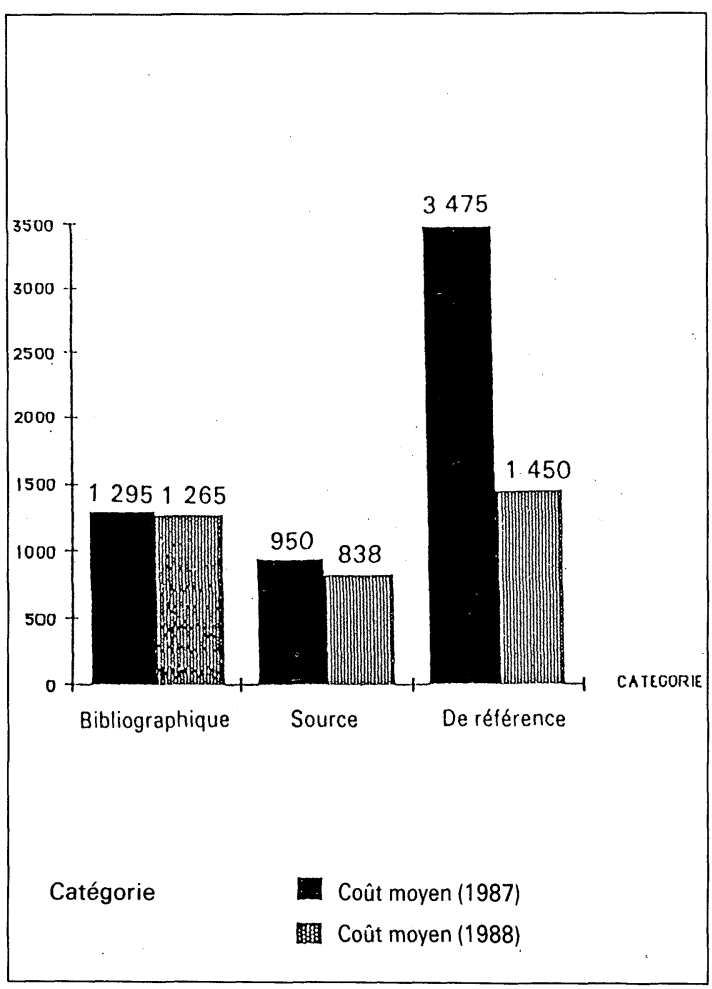


Figure 7

Bases de données sur CD-ROM : coût moyen par discipline

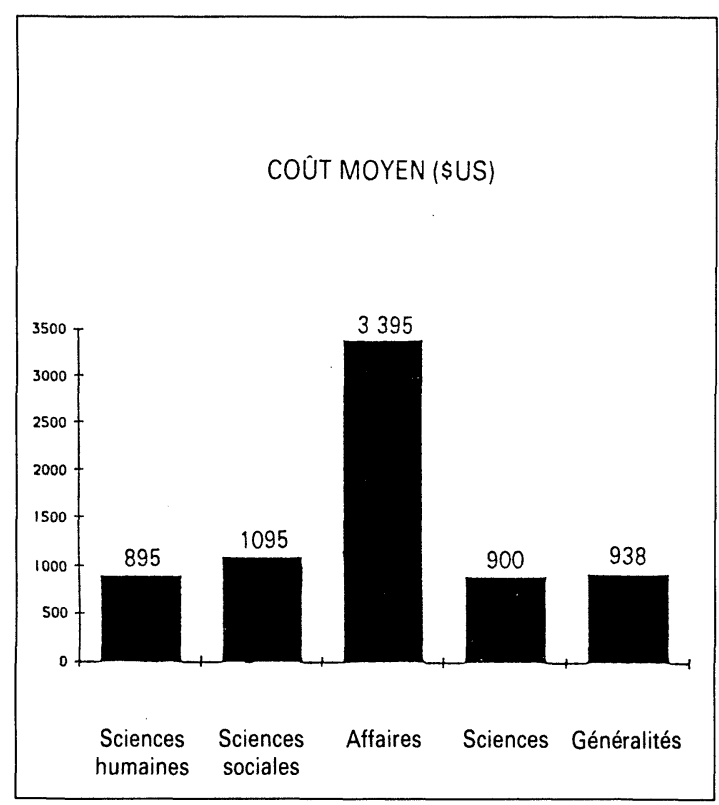

Un autre avantage du CD-ROM est qu'il permet une meilleure planification budgétaire. Contrairement à la recherche en-ligne où le volume de consultation et d'impression (souvent difficile à prévoir et à planifier) sont les facteurs déterminants pour l'allocation budgétaire, avec le CDROM, on revient au mode d'abonnement annuel fixe, similaire à celui des publications en série et familier aux bibliothèques.

Le principal désavantage des bases de données sur CD-ROM est qu'elles sont encore passablement dispendieuses. Ainsi, selon I'analyse de Paul Travis Nicholls à partir du Optical Publishing Directory ${ }^{9}$, le coût moyen des bases de données commerciales en Amérique du Nord était, en 1987, de \$ 1907 (en dollars américains). En 1988 , toutefois, ce coût était passé à $\$ 1184$, soit une diminution d'environ $37,8 \%$. On voit donc ici une nette tendance à la baisse, surtout parce qu'il commence à y avoir une certaine économie d'échelle. Cette tendance est confirmée par Stephen Arnold de UMI/Data Courrier ${ }^{10}$.
Certains serveurs, comme H.W. Wilson, annoncent même qu'en termes de revenus, leurs CDROM sont sur le point de surpasser leur service de recherche en-ligne ${ }^{11}$.

Lorsqu'on analyse l'évolution des coûts par type de contenu (fig.6), on constate que c'est surtout pour les ouvrages de référence que la baisse des coûts est la plus significative $(58,2 \%)$. Quant aux coûts par discipline, c'est sans grande surprise que l'on voit (fig.7) un coût moyen beaucoup plus élevé (3:1) des bases de données dans le domaine des affaires, probablement à cause de la nature même de l'information (statistique/numérique) qui requiert un plus grand travail de validation et à cause également de la plus grande fréquence des mises à jour.

Une analyse des 265 bases de données recensées par CD-ROM Databases sur le serveur NewsNet ${ }^{12}$ présente toutefois des résultats sensiblement différents où le coût moyen d'une base de données sur CD-ROM était, en avril 1989, de $\$ 2317$ et où l'écart des coûts entre les produits allait de $\$ 30$ à $\$ 75,000$ ! Néanmoins, I'on constate ici également que les produits les plus dispendieux sont ceux du domaine des affaires et de l'actualité (fig. 8).

Figure 8 Coût moyen par discipline
des produits sur CD-ROM

\begin{tabular}{|lccc|}
\hline & $\begin{array}{c}\text { Nbre de } \\
\text { produits }\end{array}$ & $\begin{array}{c}\text { Coût } \\
\text { moyen }\end{array}$ & Écart des prix \\
Discipline & 24 & $\$ 5915$ & $\$ 49-\$ 20400$ \\
$\begin{array}{l}\text { Affaires } \\
\text { Divers }\end{array}$ & 19 & $\$ 4793$ & $\$ 249-\$ 75000$ \\
$\begin{array}{l}\text { Sciences de la } \\
\text { santé }\end{array}$ & 35 & $\$ 2231$ & $\$ 114-\$ 7500$ \\
$\begin{array}{l}\text { Bibliothéconomie } \\
\text { et référence }\end{array}$ & 42 & $\$ 2085$ & $\$ 30-\$ 25000$ \\
$\begin{array}{l}\text { Sciences de la } \\
\text { terre }\end{array}$ & 21 & $\$ 1693$ & $\$ 49-\$ 15000$ \\
$\begin{array}{l}\text { Sciences } \\
\text { juridiques }\end{array}$ & 12 & $\$ 1260$ & $\$ 395-\$ 3000$ \\
Sciences sociales & 26 & $\$ 1237$ & $\$ 125-\$ 5000$ \\
Sciences et & 48 & $\$ 1000$ & $\$ 30-\$ 3200$ \\
techniques & & & \\
$\begin{array}{l}\text { Sciences } \\
\text { humaines }\end{array}$ & 14 & $\$ 812$ & $\$ 95-\$ 2250$ \\
\hline
\end{tabular}

9. Paul Travis Nicholls, "Information Resources on Laserdisk: Statistical Profile of Currently Available CD-ROM Database Products", Laserdisk Professional, vol. 2 no. 2 (March 1989), 101-108.

D. Stephen E. Arnold, "CD-ROM Pricing: Bound Down", Laserdisk Professional, vol. 2, no. 2 (March 1989), 6-10.
11. Datapro Research Corp, Directory of Online Service. Delron, N.J., McGraw-Hill, 1989, p.OL20-450-101 (July 1989).

12. CD-ROM Databases.. 


\section{Marché du CD-ROM}

Selon une étude de Frost et Sullivan ${ }^{13}$, environ $15 \%$ des producteurs de bases de données et des serveurs américains ont des produits sur CDROM. La plupart montrent encore une attitude typique de conservatisme d'anticipation. Comme le faisait remarquer l'un des producteurs de bases de données sur CD-ROM, interrogé dans le cadre de la présente étude:

We look upon this first edition of (...) on CDROM as an experiment. We want to test the market, and allow our users some input into development before leaping into $C D$ production with both feet. (...) CD sales have been quite disappointing, for a variety of reasons... ${ }^{14}$

On peut analyser le marché des supports optiques, et du CD-ROM en particulier, en termes des baromètres ou indicateurs suivants :

1) Les marchés concurrents (papier, ordinolingue, microforme)

À cet égard, malgré une légère percée prévue pour le support optique d'ici 1991, ce dernier demeurera encore très marginal par rapport au papier comme support de stockage de l'information (fig. 9). Toutefois, certaines études comparatives ${ }^{15}$ montrent des coûts (ou investissements) moyens par recherche significativement plus bas pour le CD-ROM $(\$ 4.61)$ que pour la recherche en-ligne (\$6.44). Un tel facteur pourrait changer considérablement les données.

\section{2) Le nombre et les catégories de lecteurs vendus}

Le nombre de lecteurs vendus croît de façon soutenue depuis 1987 et les prévisions demeurent encore assez optimistes aussi bien en Amérique du Nord (fig. 10) qu'en Europe (fig. 11).

Quant aux types de lecteurs vendus, l'on prévoit (fig. 12) qu'à partir de cette année déjà, les lecteurs de CD-WORM (write-once-read-many) dépasseront ceux de CD-ROM, sans compter les nouveaux produits optiques (CDI, DVI, etc.) qui sont à être mis au point et qui ne sont pas encore apparus comme des facteurs significatifs du marché 16 .
Figure 9

Distribution du marché des supports de stockage d'information

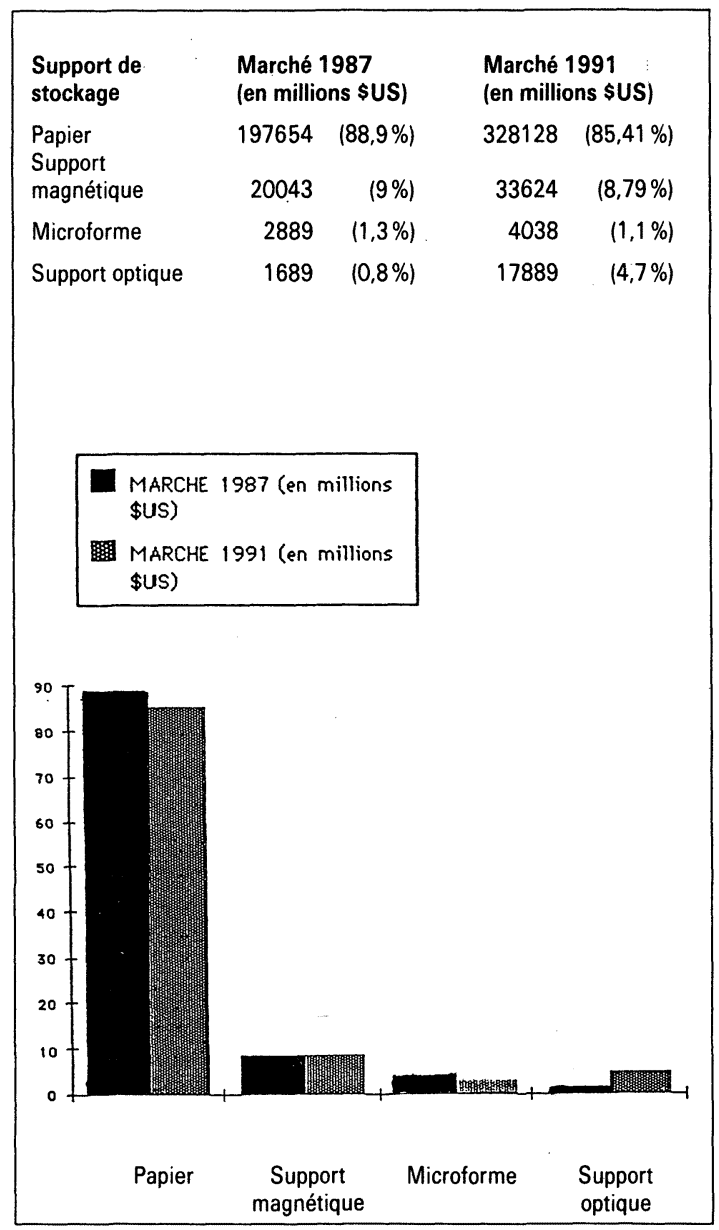

\section{3) L'ampleur et la distribution des parcs de lecteurs et de produits CD-ROM vendus}

Selon Evans Research Corporation 17 (fig. 13), le marché du CD-ROM au Canada est appelé à croître considérablement entre 1986 et 1990 (avec un seuil de croissance remarquable en 1989), tant en termes de parcs de lecteurs que de celui des disques installés.
13. Frost and Sullivan, The US Market for Online Databases. Winter 1988-89.

14. Correspondance privée.

15. Hollis Landrum, «Determining and Recovering CD-ROM Search Costs", Laserdisk Professional, vol. 2 no. 2 (March 1989), 23-25.
17. Evans Research Corporation, EDP In-Depth Report, vol. 15, no. 9 (September 1986), 13.

16. Susan Motley, "CD-ROM : 1988 in Review »... 
Figure 10

Lecteurs de CD-ROM vendus (États-Unis et Canada)

\begin{tabular}{|cc|}
\hline Année & Lecteurs de CD-ROM Vendus \\
1987 & 65000 \\
1988 & 149000 \\
1989 & 296000 \\
1990 & 470000 \\
1991 & 724000 \\
\hline
\end{tabular}

\section{4) Les équipements de lecture}

Dans les bibliothèques, selon l'enquête annuelle d'OCLC de 198818, c'est Hitachi qui occupait la majeure partie $(40,8 \%)$ du parc des lecteurs installés (fig. 14).

\section{5) Les créneaux d'application}

Selon Rotchild Consultants, les bases de données constitueront, en 1990 , quelque $36 \%$ du marché d'application des produits CD-ROM (fig. 15).

Figure 11

Le parc de lecteurs CD-ROM

en Europe (en unités)

\begin{tabular}{|lrrrrrr|}
\hline Pays & & & & & & \\
& 1987 & 1988 & 1989 & 1990 & 1991 & 1992 \\
Royaume-Uni & 720 & 2400 & 6400 & 17200 & 32500 & 54000 \\
$\begin{array}{l}\text { France } \\
\text { Allemagne }\end{array}$ & 420 & 2150 & 5300 & 12800 & 27500 & 45500 \\
de l'ouest & 720 & 2950 & 7200 & 18000 & 36000 & 61000 \\
Italie & 4020 & 13800 & 19600 & 27300 & 37000 & 45000 \\
$\begin{array}{l}\text { Scandinavie } \\
\text { Autres pays }\end{array}$ & 200 & 850 & 2400 & 9300 & 16500 & 26000 \\
d'Europe & 620 & 2950 & 6300 & 15900 & 32500 & 52500 \\
Total & 6700 & 25100 & 47200 & 100500 & 182000 & 284000 \\
& & & & & & \\
\hline
\end{tabular}


Figure 12

Marché des

lecteurs optiques (1987-1991)

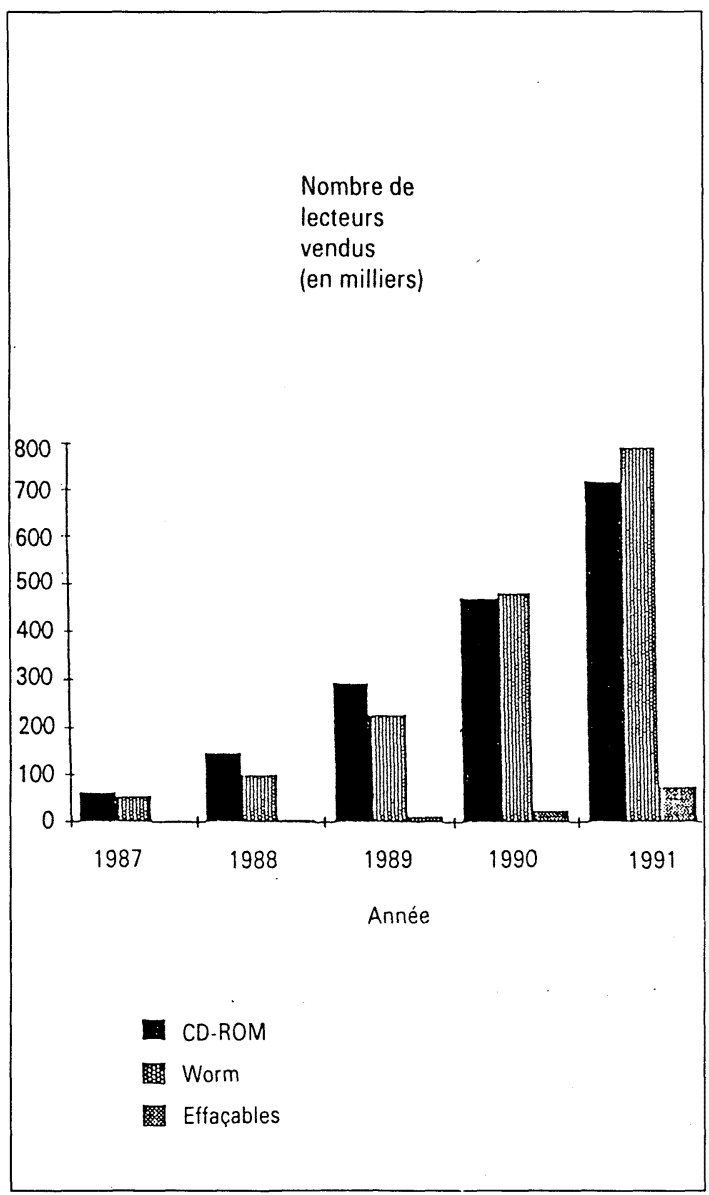

Figure 13

Marché du CD-ROM au Canada (1986-1990)

\begin{tabular}{|ccc|}
\hline Année & Lecteurs de CD-ROM & Disques CD-ROM \\
& & \\
1986 & 300 & 900 \\
1987 & 2000 & 2500 \\
1988 & 7500 & 8000 \\
1989 & 20000 & 22000 \\
1990 & 30000 & 43000 \\
& & \\
\hline
\end{tabular}

Figure 14

Lecteurs de CD-ROM dans les bibliothèques

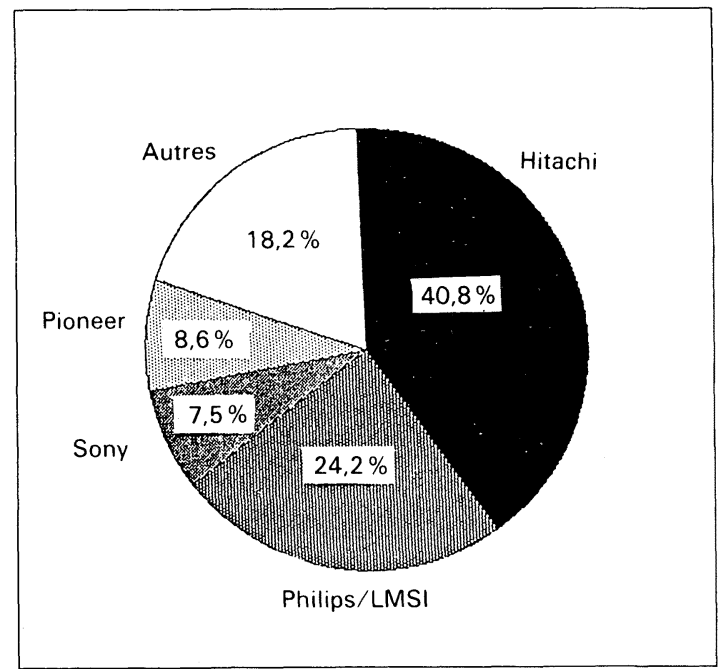

Figure 15

Distribution des applications du CD-ROM (Prévisions 1990)

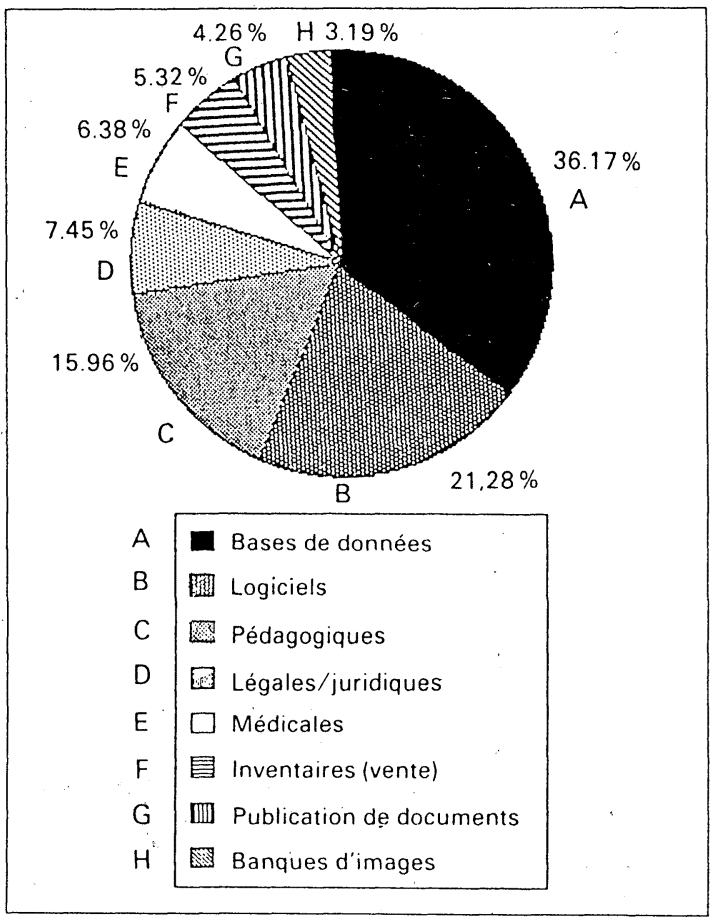




\section{6) La structure du marché et la répartition des revenus parmi les intervenants}

Une étude de Learned Information ${ }^{19}$ affirme que le marché américain de l'information en-ligne, un marché de plus de \$6 milliards, a commencé, dès 1988, à ressentir l'impact du CD-ROM considéré comme une «menace " pour ce marché.

Si on compare la structure du marché du CDROM (fig.17) à celle du marché de la recherche en-ligne (fig.16), on peut faire les observations suivantes:

a) contrairement au marché de la recherche enligne, celui du CD-ROM n'a ni serveurs, ni réseaux de télécommunications; il y a donc une plus grande autonomie locale et une complète liberté de consultation ; b) les serveurs sont remplacés, dans le marché du CD-ROM, par les producteurs et distributeurs de disques, qui sont assez diversifiés: producteurs de bases de données (ex. University Microfilms International), serveurs (ex. Dialog), producteurs de disques (ex. SilverPlatter);

c) le marché du CD-ROM s'adresse aussi bien aux centres de courtage (ex. bibliothèques) qu'aux utilisateurs eux-mêmes principalement à cause de la convivialité de l'interrogation; toutefois, l'investissement requis (équipement, abonnement aux disques) représente encore un frein majeur au développement du marché grand-public;

Figure 16

Structure du marché : recherche en-ligne

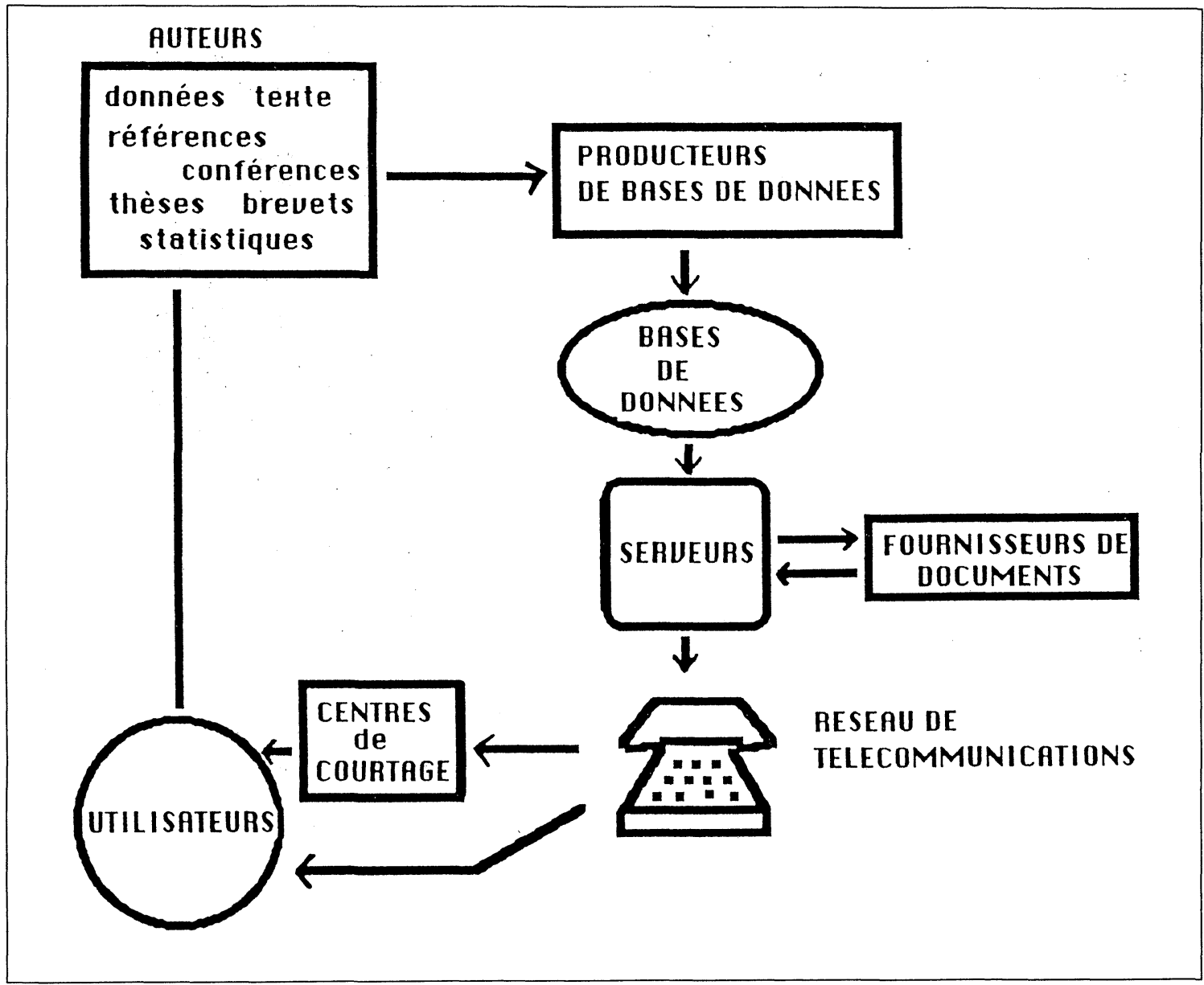

19. The CD-ROM and the Online Database Markets, Medford, N.J., Learned Information, 1988. 
Figure 17

Structure du marché : CD-ROM

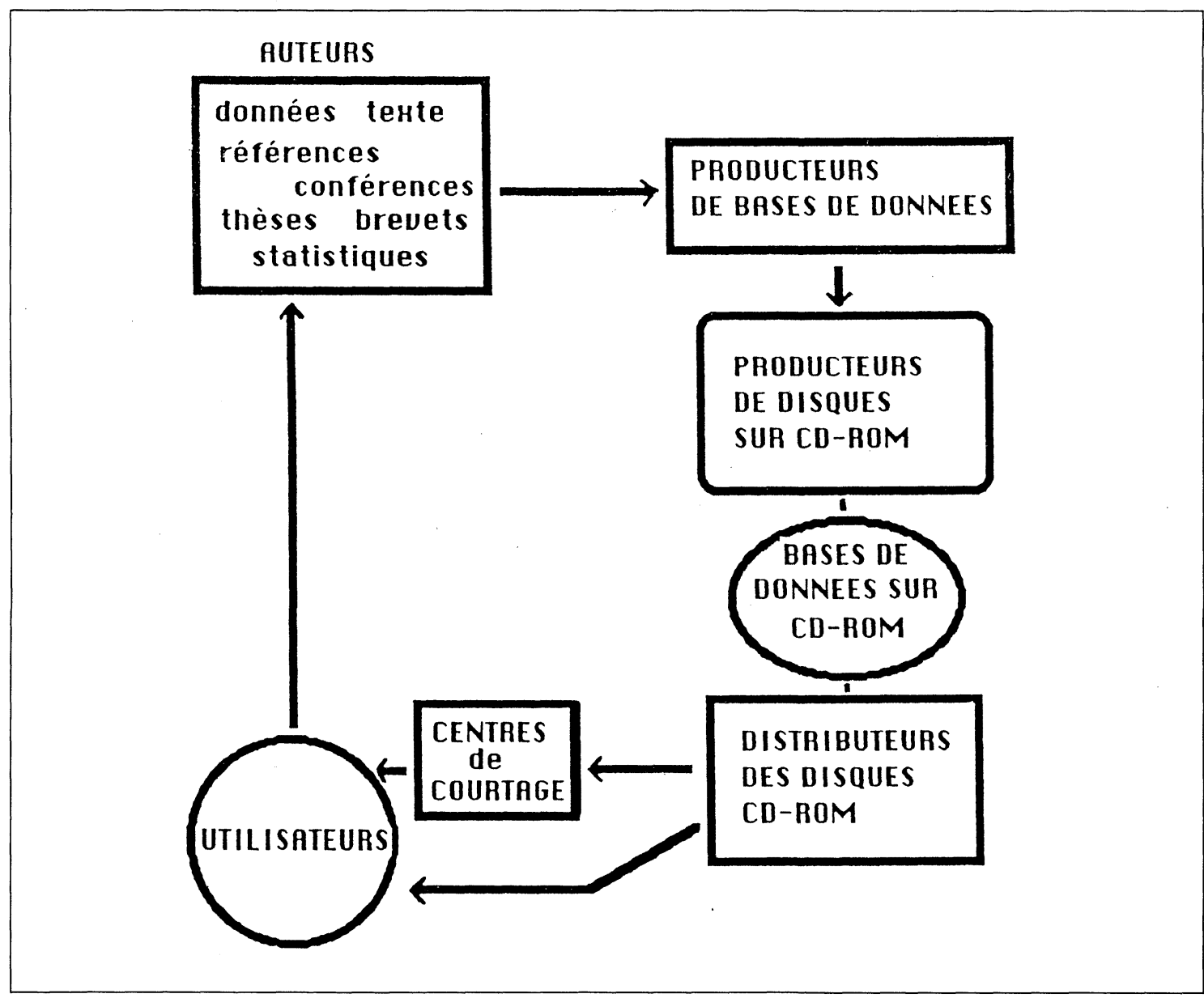

d) contrairement au marché de la recherche enligne, il n'y a pas, dans le marché du CD-ROM, de fournisseurs de documents (surtout pour les bases de données bibliographiques); un document identifié lors d'une recherche sur CD-ROM doit donc être commandé en-ligne, dans une seconde étape (quoique certains serveurs - ex. Wilsonline - permettent de passer du CD-ROM à la recherche en-ligne assez facilement), ou selon la procédure habituelle de la bibliothèque (acquisitions, PEB);

e) les figures 18 et 19 présentent une comparaison des deux marchés en termes de répartition des revenus générés parmi les intervenants. Le gros des revenus, dans le marché de la recherche en-ligne, va donc aux serveurs ou distributeurs de bases de données $(56,1 \%$ en 1984 et $53,8 \%$ en 1989$)$. Quant aux réseaux de télécommunications, leur part du marché est passée de $22,3 \%$ en 1984 à $10,8 \%$ en 1989). Les producteurs de bases de données qui sont, de fait, à l'origine de tout le processus, doivent se contenter d'à peine $20,2 \%$ des revenus (28,9\% prévus en 1989). Par ailleurs, avec le marché du CD-ROM, le processus est passablement différent. D'abord, comme on l'a vu, il n'existe ni serveurs, ni réseaux de télécommunications. Les nouveaux acteurs sont principalement l'éditeur et le fabricant de disques. Mais ce qui est surtout intéressant dans ce nouveau marché, c'est la place prépondérante du producteur des bases de données, dont la part des revenus était de $32 \%$ en 1987 et qui sera de quelque $45 \%$ en 1992. C'est pourquoi d'aucuns ont parlé des CD-ROM comme de la «revanche des producteurs de bases de données".

\section{Le marché des bibliothèques}

Au printemps de 1988, David Fox a effectué, pour le CD-ROM Interest Groupe de la Canadian Library Association, une enquête auprès de $\mathbf{3 7 6}$ 
bibliothèques de tout le Canada pour «compiler de l'information sur les expériences d'implantation du CD-ROM» ${ }^{20}$.

Figure 18

Répartition des revenus de I'I.I.E.* (1984,1989)

Répartition des revenus de l'I.I.E. parmi ses intervenants, États-Unis, 1984 (en millions de dollars)

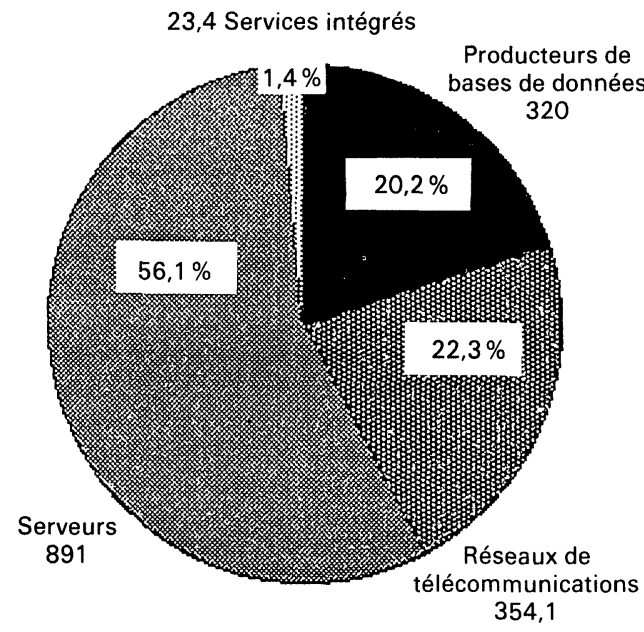

Répartition des revenus de I'I.I.E. parmi ses intervenants, États-Unis, 1989 (en millions de dollars)

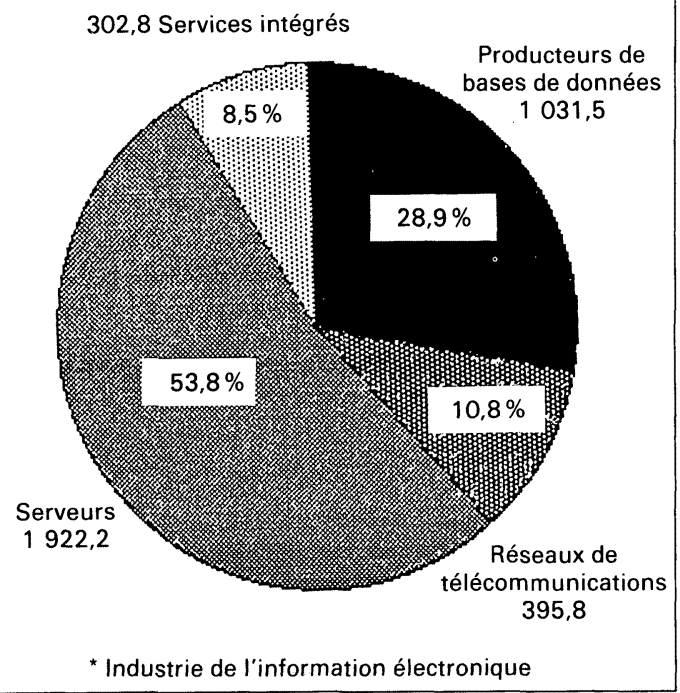

Figure 19

Distribution du marché du CD-ROM (1987-1992)

\begin{tabular}{|c|c|c|}
\hline Intervenants & $\begin{array}{l}\text { \% du marché } \\
\text { (1987) }\end{array}$ & $\begin{array}{l}\% \text { du ma } \\
\quad 199\end{array}$ \\
\hline $\begin{array}{l}\text { Conception des bases de } \\
\text { données }\end{array}$ & 32 & 45 \\
\hline $\begin{array}{l}\text { Production des bases sur } \\
\text { disques }\end{array}$ & 18 & 40 \\
\hline Intégration de systèmes & 20 & 3 \\
\hline Fabrication des disques & 5 & 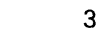 \\
\hline Fabrication des lecteurs & 10 & 5 \\
\hline $\begin{array}{l}\text { Fabrication des systèmes } \\
\text { d'appoint }\end{array}$ & 15 & \\
\hline
\end{tabular}

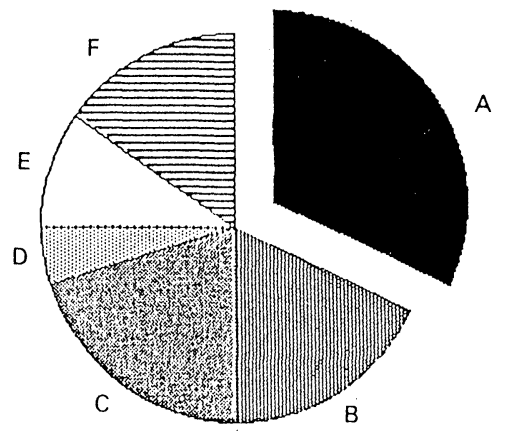

A Conception des bases de données

B 闻 Production des bases sur disques

C Intégration de systèmes

D Fabrication des disques

E $\square$ Fabrication des lecteurs

F 目 Fabrication des systèmes d'appoint

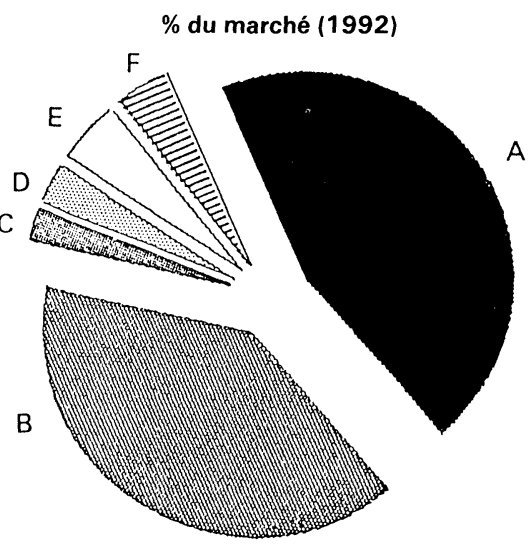


L'enquête couvrait tous les types de bibliothèques. Nous commenterons les principaux résultats de cette enquête.

- 25,6\% des bibliothèques avaient implanté un service de CD-ROM, la presque totalité dans le Canada anglophone; l'absence presque totale de CD-ROM au Québec était due surtout à l'absence de produits en langue française ;

- le taux d'implantation le plus élevé, 65,7\%, venait des bibliothèques universitaires;

- I'utilisation principale des CD-ROM était : outil d'aide au catalogage $(43,3 \%)$; bases de données bibliographiques ( $25 \%)$; outil d'aide aux acquisitions $(17,3 \%)$;

- $87,1 \%$ des services de CD-ROM avaient moins de un an et demi ;

- les principales raisons évoquées pour l'utilisation du CD-ROM étaient: I'amélioration du service $(30,3 \%)$; le remplacement de l'imprimé ou de la recherche en-ligne $(24,4 \%)$; l'accroissement de la productivité $(18,5 \%)$.

- $82,9 \%$ des bibliothèques ayant implanté un service de CD-ROM considèrent ce projet comme une réussite (totale ou partielle); $64,3 \%$ de ces bibliothèques disent vouloir étendre le service;

- enfin, $38 \%$ des bibliothèques $141,6 \%=$ anglophones, $26,1 \%=$ francophones) n'ayant pas de service de CD-ROM disaient vouloir en implanter un avant deux ans.

\section{Enquête sur les CD-ROM dans les bibliothèques du Québec ${ }^{21}$}

En avril 1989, une enquête a été effectuée auprès de toutes les bibliothèques et centres de documentation du Québec $(n=801)$ pour évaluer le degré de pénétration du CD-ROM dans ces milieux. L'enquête de David Fox ${ }^{22}$, pour la Canadian Library Association, un an plus tôt, avait déjà indiqué une absence presque totale du CDROM dans les bibliothèques québécoises.

L'enquête postale a été réalisée entre le 13 mars et le 7 avril 1989 (voir le questionnaire en annexe). Au total, 373 questionnaires ont été retournés, pour un taux de réponse de $46,5 \%$ (fig.20). Pour fins d'analyse, les répondants ont été regroupés en quatre catégories: publiques (municipales, BCP); universitaires; collégiales et scolaires; spécialisées (spécialisées, gouvernementales, centres de documentation).

Nous avons d'abord cherché à savoir (question 2) si les répondants possédaient un service de téléréférence. Nous avions, en effet, émis I'hypothèse d'une corrélation entre l'existence d'un service de téléréférence et l'implantation d'un service de CD-ROM pour la recherche d'information. Comme on peut le constater dans la fig. 21 , près de la moitié $(48,5 \%)$ des répondants possèdent un service de téléréférence. Ce service est surtout implanté dans les bibliothèques spécialisées $(59,5 \%)$, les bibliothèques publiques faisant un peu figure de parent pauvre $(10,3 \%)$.

Une question complémentaire (question 3) visait à déterminer les serveurs les plus utilisés dans les services de téléréférence. C'est Dialog qui vient en tête de liste, non seulement pour l'ensemble des bibliothèques, mais également pour chaque catégorie (fig.22). Viennent ensuite, dans l'ordre, IST Informathèque (avant qu'elle ne devienne Prima Télématique), CAN/OLE, Infoglobe, QL Systems, Infomart, NLM, Orbit, BRS et SDM. II est probable que ce dernier serveur soit davantage sollicité s'il prend la relève de IST Informathèque pour la diffusion des bases de données québécoises. Quant à UTLAS, c'est un serveur de nature un peu différente (catalographique plutôt que bibliographique) qui est rarement utilisé, de façon intensive, pour préparer des bibliographies pour des utilisateurs.

Les questions suivantes (questions 4,5 et 6 ) visaient à déterminer le nombre de répondants ayant déjà implanté un service de CD-ROM, ceux qui prévoyaient en implanter un dans un proche avenir, et les bases de données sur CD-ROM les plus utilisées. Un an après l'enquête de $\mathrm{Fox}^{23}$, force nous est de constater que le Québec accuse toujours un sérieux retard sur le reste du Canada et les États-Unis en ce qui concerne l'implantation de services de CD-ROM dans les bibliothèques. En effet, seulement $7,7 \%$ des répondants ( 29 sur 373 ) disent offrir un service de CD-ROM à leur clientèle (fig.23). Ici encore, ce sont les biblio-

21. Cette enquête a été préparée avec la collaboration de Louise Rivard, Louise Deschênes et Caroline Robillard, alors étudiantes à I'EBSI, dans le cadre du cours BLT 6206 (Recherche sur la communication de l'information). 
Figure 20

Enquête sur le CD-ROM au Québec : Nombre de répondants

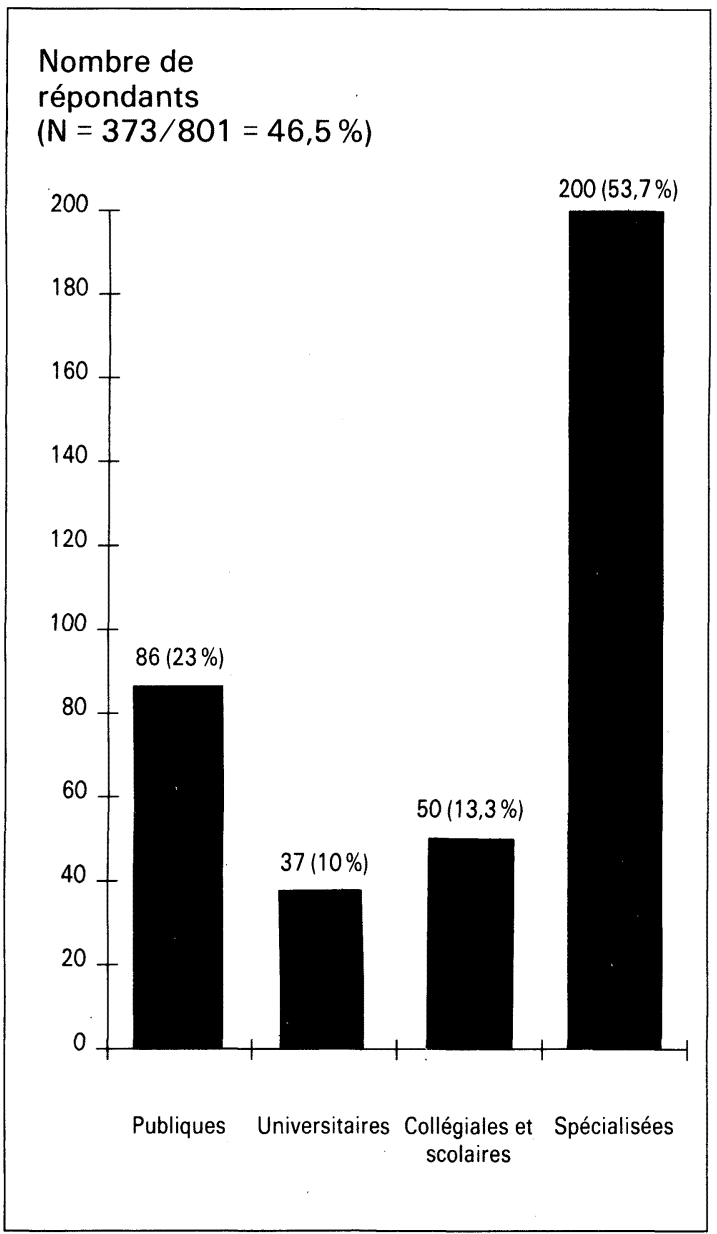

Figure 21

Bibliothèques ayant un service de téléréférence

\begin{tabular}{|ll} 
Bibliothèques & Nombre $(\%)$ \\
Publiques & $9 / 86(10,3 \%)$ \\
Universitaires & $32 / 37(86,5 \%)$ \\
Collégiales et scolaires & $21 / 50(42 \%)$ \\
Spécialisées & $119 / 200(59,5 \%)$ \\
& \\
Total & $181 / 373(48,5 \%)$
\end{tabular}

Figure 22

Serveurs utilisés dans les bibliothèques du Québec

\begin{tabular}{|c|c|c|c|c|c|}
\hline Serveurs & $\begin{array}{l}\text { Bibl. } \\
\text { publ. }\end{array}$ & $\begin{array}{l}\text { Bibl. } \\
\text { univ. }\end{array}$ & $\begin{array}{l}\text { Bibl. collég. } \\
\text { et scol. }\end{array}$ & $\begin{array}{c}\text { Bibl. } \\
\text { spécial. }\end{array}$ & Tota \\
\hline DIALOG & 5 & 28 & 13 & 86 & 132 \\
\hline CAN/OLE & 3 & 17 & 4 & 54 & 78 \\
\hline BRS & & 12 & & 11 & 23 \\
\hline INFOMART & 1 & 3 & 1 & 32 & 37 \\
\hline WILSONLINE & 2 & 10 & & 5 & 17 \\
\hline DOW JONES & & & & 9 & 9 \\
\hline VUTEXT & & & & 1 & 1 \\
\hline INFOGLOBE & 2 & 6 & 1 & 33 & 42 \\
\hline NLM & & 5 & & 25 & 30 \\
\hline ORBIT & & 10 & & 17 & 27 \\
\hline MEAD DATA & & 10 & & 7 & 17 \\
\hline SDM & 4 & 3 & 4 & 11 & 22 \\
\hline IST/QUESTEL & 5 & 15 & 9 & 54 & 83 \\
\hline INFOLINE & & 3 & & 4 & 7 \\
\hline OL SYSTEMS & & 9 & & 29 & 38 \\
\hline UTLAS & 4 & 16 & 5 & 19 & 44 \\
\hline AUTRES & 5 & 24 & 11 & 79 & 119 \\
\hline
\end{tabular}

$\begin{array}{lr}\text { Autres serveurs } & \mathbf{f} \\ & \\ \text { SOQUIJ } & 12 \\ \text { STN } & 11 \\ \text { DOBIS } & 10 \\ \text { FINANCIAL POST } & 10 \\ \text { DATA STAR } & 7 \\ \text { CAN/LAW } & 7 \\ \text { RENARD } & 6 \\ \text { INFOPUQ } & 5 \\ \text { BTQ (OLF) } & 5 \\ \text { CRDI } & 4 \\ \text { BADADUQ } & 4 \\ \text { NEWSNET } & 3 \\ \text { WESTLAW } & 3 \\ \text { CANADIAN TAX ONLINE } & 3 \\ \text { DUNSERVE } & 3 \\ \text { COMPUSERVE } & 3 \\ \text { ESA-IRS (EUROLINE) } & 2 \\ \text { 19 SERVEUrS } & 1 \\ & \end{array}$

Figure 23

Bibliothèques ayant un ou des CD-ROM

$\begin{array}{ll}\text { Bibliothèques } & \text { Nombre }(\%) \\ \text { Publiques } & 3 / 86(3,4 \%) \\ \text { Universitaires } & 11 / 37(30,5 \%) \\ \text { Collégiales et scolaires } & 0 / 50 \\ \text { Spécialisées } & 15 / 200(7,5 \%) \\ & \\ \text { Total } & 29 / 373(7,7 \%)\end{array}$


Figure 24

Enquêtes sur le CD-ROM

\begin{tabular}{|c|c|c|c|c|c|c|c|}
\hline Date & $\begin{array}{l}\text { Huang }{ }^{24} \\
88\end{array}$ & $\begin{array}{l}\text { Solomon } 25 \\
09-87\end{array}$ & $\begin{array}{l}\text { Wong } \\
\text { 26 } \\
02-88\end{array}$ & $\begin{array}{l}\text { Fox }{ }^{27} \\
88\end{array}$ & $\begin{array}{l}\text { Herter }^{28} \\
\quad 88\end{array}$ & $\begin{array}{l}\text { Chen } 29 \\
07-87\end{array}$ & $\begin{array}{l}\text { Deschatelets } \\
04-89\end{array}$ \\
\hline $\begin{array}{l}\text { Nombre de questionnaires } \\
\text { envoyés }(n)\end{array}$ & 51 & 150 & 16 & 376 & 448 & 720 & 801 \\
\hline $\begin{array}{l}\text { Taux de réponse } \\
\% \text { ayant service de CD- } \\
\text { ROM }\end{array}$ & $\begin{array}{l}100 \% \\
100 \%\end{array}$ & $\begin{array}{l}80 \% \\
68 \%\end{array}$ & $\begin{array}{l}100 \% \\
68,7 \%\end{array}$ & $\begin{array}{l}72,6 \% \\
25,6 \%\end{array}$ & $\begin{array}{l}n / a \\
44 \%\end{array}$ & $\begin{array}{l}40 \% \\
30 \%\end{array}$ & $\begin{array}{l}46,5 \% \\
7,7 \%\end{array}$ \\
\hline
\end{tabular}

Figure 25

Bases de données sur CD-ROM

\begin{tabular}{|lc|}
\hline Bases de données & $\mathbf{f}$ \\
CCINFOdisc & 7 \\
MEDLINE & 7 \\
ABI/INFORM & 5 \\
ERIC & 4 \\
CBCA & 4 \\
BOOKS IN PRINT + & 3 \\
PSYCHLIT & 3 \\
WILSONDISC & 3 \\
BIBLIOFILE & 2 \\
MICROSOFT BOOKSHELF & 2 \\
23 bases de données & 1 \\
& TOTAL $\mathbf{N}=\mathbf{3 3}$ \\
& $\mathbf{f = 6 3}$ \\
\hline
\end{tabular}

thèques universitaires et les bibliothèques spécialisées qui font figure de pionnières, les autres bibliothèques étant presque totalement absentes de ce marché.

Ces chiffres sont nettement inférieurs à ceux de toutes les autres enquêtes effectuées à date sur les CD-ROM, comme on peut le constater dans la fig. 24.

Si on analyse la distribution des 29 services québécois par type de bibliothèque, on constate que les bibliothèques spécialisées occupent la plus grande part de ce "marché " $(51,8 \%)$, suivies des bibliothèques universitaires $(37,9 \%)$ et des bibliothèques publiques $(10,3 \%)$. Cette distribution est assez similaire à celle des enquêtes précédentes ${ }^{30}$. Évidemment, le nombre restreint de bibliothèques offrant ce service (29) ne permet pas de parler véritablement de "marché», ni de généraliser sans danger sur les tendances et orientations de ce nouveau service. Néanmoins, il est intéressant d'analyser plus en détail ces 29 services de CD-ROM. D'autant plus que 15,4\% des répondants $(n=54)$ qui $n$ 'ont pas de service de CD-ROM prévoient en implanter un dans un proche avenir.

L'hypothèse d'une corrélation entre le service de téléréférence et le service de CD-ROM semble se confirmer. En effet, des 29 bibliothèques qui offrent un service de CD-ROM, 27 offrent également un service de téléréférence. En outre, la plupart de ces services de téléréférence sont assez importants puisque $17 \mathrm{~d}$ 'entre eux offrent accès à 5 serveurs ou plus. Quant aux 54 répondants qui prévoient offrir un service de $C D$ ROM dans un proche avenir, ils se distribuent de la façon suivante : 13 bibliothèques publiques, 13 bibliothèques universitaires, 22 bibliothèques spécialisées et 6 bibliothèques de collège. Les deux tiers $(36 / 54)$ offrent déjà un service de téléréférence.

En ce qui concerne les produits ou bases de données sur CD-ROM les plus populaires, ce sont, par ordre décroissant (fig.25): Medline et CCINFOdisc, ABI/INFORM, ERIC, CBCA et BIP+31. II est à remarquer que les produits mentionnés sont passablement diversifiés $(n=33)$ et qu'aucun n'a encore véritablement démontré une grande ferveur populaire (pour Medline et CCINFOdisc,
24. Samuel T. Huang, "CD-ROM Databases: Unique Resource for Libraries", Laserdisk Professional, vol. 2, no. 2 (March 1989), 62-67.

25. Kristine Solomon, "The impact of CD-ROM on Reference Departments", $R Q$, vol. 28, no. 2 (Winter 1988), 203-219.

26. Maureen Wong et al., "A Survey on the Use of CD-ROM in Canadian Medical School Libraries", Laserdisk Professional, vol. 2, no. 1 (January 1989), 51-58.
27. David Fox, «CD-ROM Use in Canadian..

28. Nancy K. Herter, «1988 OCLC Survey...

29. Ching-chih Chen, "CD-ROM Survey in American Academic \& College Libraries", in 11th International Online Information Meeting, London, 8-10 December 1987 Medford, N.J., Learned Information, 1987, p. 9-12.

30. Notes 25 à 29 et Reese, "Surveying the CD-ROM Scene: What's out There? ", in Online ' 88 Conference Proceedings, New York, October 10-12, 1988, Weston, CT, Online Inc., 1988, p. 132-136.

31. Le CD-ROM «Choix», de SDM, n'était pas encore disponible au moment de l'enquête. 
$f=7)$. Cela corrobore les résultats de l'étude de Link Resources ${ }^{32}$ selon lesquels il n'existe pas encore de produit "best-seller» en trois ans d'existence du CD-ROM, l'encyclopédie Grolier ${ }^{33}$ étant le meilleur vendeur aux États-Unis avec à peine 3000 copies.

Ce sont encore Medline et CCINFOdisc qui sont les produits sur CD-ROM les plus utilisés (fig.26), suivis de $A B I / I N F O R M, C B C A, B I P+$ et ASFA. Encore là, le volume des données recueillies ne permet pas d'analyse très détaillée.

La question suivante (question 7) visait à déterminer le nombre de lecteurs de CD-ROM utilisés par les bibliothèques ayant implanté un service. La grande majorité des répondants (21/ 29) ne possèdent qu'un seul lecteur, ce qui montre un service encore en stade d'implantation ou alors un service un peu marginal avec peu de produits offerts et relativement peu d'utilisateurs (fig.27).

Les questions 8 à 12 voulaient connaître un peu mieux l'attitude des répondants ayant implanté un service de CD-ROM quant aux raisons qui les avaient incités à choisir le CDROM (fig.28), les utilisateurs visés (fig.29), la politique de tarification du service (fig.30), certaines observations engendrées par l'utilisation du CD-ROM (fig.31) et les problèmes encourus (fig.32).

Dans l'ensemble, on remarque que la majorité des répondants ont choisi le CD-ROM d'abord à cause du coût fixe des recherches, ensuite parce qu'il permet l'interrogation par les usagers euxmêmes et parallèlement, à cause de la facilité d'utilisation des équipements; cette distribution, toutefois, varie un peu selon le type de bibliothèque (fig. 28); les utilisateurs sont, dans l'ordre, les bibliotechniciens, les bibliothécaires, les usagers (avec assistance) et les usagers (sans assistance); cette distribution est assez caractéristique d'un service en pleine implantation; pour la grande majorité des services $(75,6 \%)$, les recherches sont gratuites; plusieurs répondants ont mentionné que leur politique actuelle est à l'essai.

L'enquête démontre également que le CD-ROM est très favorablement accepté par les usagers $(100 \%)$ et attire de nouveaux usagers $(73,1 \%)$; toutefois, malgré ses prétentions, il est assez difficile à utiliser par les usagers eux-mêmes $(69,2 \%)$ et il occasionne plus de travail au personnel de la bibliothèque $(76,9 \%)$; on ne le confond qu'assez peu avec le catalogue de la bibliothèque $(42,3 \%)$.
Le CD-ROM occasionne des problèmes d'installation (68\%), met en évidence un manque de familiarité avec le micro-ordinateur $(56 \%)$ et entraîne des difficultés, chez les utilisateurs, avec les stratégies de recherche (64\%) et la logique de recherche $(60 \%)$; par contre, il ne semble y avoir que peu de problèmes avec la documentation, le choix des termes de recherche et la visualisation des résultats.

\section{Conclusion}

Malgré les prévisions optimistes et les promesses d'un marché florissant, le CD-ROM commence à peine à montrer les signes encourageants d'un véritable marché. Aux États-Unis, il y avait un parc d'environ 88000 lecteurs de CD-ROM à la fin de 1988 et l'on en prévoyait un de 220000 pour la fin de 1989 (bien que plusieurs croient toujours ce chiffre exagéré) ${ }^{34}$. Au Canada, on parle d'à peine 7500 lecteurs en 1988 et de 20,000 pour $1989^{35}$. Pourquoi ce retard dans une technologie que l'on prétend parfaite pour les marchés de masse? Trois raisons ont été avancées: 1) les prix élevés pour l'équipement (coût moyen de $\$ 1000$ pour un lecteur comparé au lecteur de $C D$-audio qui se vend pour à peine plus de $\$ 250$ ) et les produits; 2) le type et la structure des contenus qui ne sont pas encore véritablement adaptés aux énormes capacités et à la flexibilité des supports (texte, son, image); 3 ) la désuétude de l'information et l'impossibilité d'effacer l'information enregistrée sur les disques (quoique l'on commence à voir apparaître des disques effaçables).

Dans les bibliothèques, on a fait montre de prudence, au début, face à l'arrivée des CD-ROM mais le marché commence à s'implanter. Selon Nancy Melin Nelson, éditeur du CD:ROM Librarian, il y avait un parc de quelque 1500 lecteurs de CD-ROM dans les bibliothèques américaines en 1988 , ce qui représenterait plus de $17 \%$ de tous les lecteurs installés. Une enquête de Research Publications ${ }^{36}$ à l'été 1987 montrait que sur les 20443 bibliothécaires et professionnels de l'information ayant répondu au questionnaire, $23,8 \%$ utilisaient déjà un CD-ROM ou l'utiliseraient avant la fin de 1987 et plus de $50 \%$ des répondants prévoyaient en utiliser un avant 1990 .

Ce sont les bibliothèques universitaires et les bibliothèques spécialisées qui sont de loin les plus gros utilisateurs des CD-ROM et elles exploitent surtout les bases de données bibliographiques et les ouvrages de référence. II est intéressant de noter que pour plusieurs bibliothèques, le $C D$ -
32. "Link Resources Corporation", in 10th International Online Meeting, London, 2-4 December, 1986, Medford, N.J., Learned Information, 1986, p. 263-269.

33. Academic American Encyclopedia.
34. "Link Resources Corporation »...

35. Evans Research Corporation, EDP In-Depth Report..

36. Information Hotline, vol. 20, no. 3 (March 1988), 3. 
Figure 26

Bases de données sur CD-ROM les plus souvent utilisées

\begin{tabular}{|lc|}
\hline Bases de données & Nombre de points \\
MEDLINE & 21 \\
CCINFOdisc & 13 \\
ABI/INFORM & 11 \\
CBCA & 9 \\
BOOKS IN PRINT+ & 7 \\
ASFA & 6 \\
WILSONDISC & 6 \\
ERIC & 5 \\
PSYCHLIT & 5 \\
BIBLIOFILE & 3 \\
DOW JONES & 3 \\
LOTUS ONE SOURCE & 3 \\
PAIS & 3 \\
NIOSHTIC & 3 \\
HSELINE & 3 \\
CISDOC & 2 \\
OXFORD ENGLISH DICT. & 2 \\
COMPUTER LIBRARY & 2 \\
NTIS & 2 \\
COMPACT AIDS & 2 \\
SCISEARCH & 2 \\
TERMIUM & 2 \\
FINANCIAL PACKAGE & 1 \\
DISSERTATION ABSTR. & 1 \\
NEWS PACKAGE & 1 \\
MOODY'S & 1 \\
& \\
LEGENDE & \\
Rang 1 = 3 points & \\
Rang 2 = 2 points & \\
Rang 3 = 1 point & \\
\hline
\end{tabular}

Figure 27

Distribution des lecteurs de CD-ROM

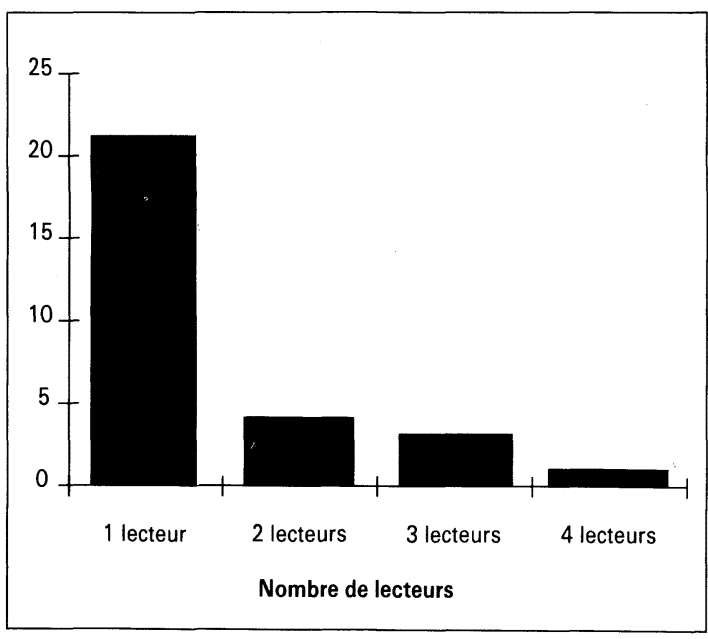

Figure 28

Raisons du choix du CD-ROM

\begin{tabular}{|lcccc|}
\hline & \multicolumn{4}{c|}{ Rangs } \\
Raison & B. Publ. & B. Univ. & B. Spécial. & Ensemble \\
Facilité d'utilisation & 2 & 3 & 3 & 3 \\
$\begin{array}{l}\text { Interrogation par les } \\
\text { usagers eux-mêmes }\end{array}$ & 4 & 2 & 2 & 2 \\
$\begin{array}{l}\text { Coûts fixes des } \\
\text { recherches }\end{array}$ & 3 & 1 & 1 & 1 \\
$\begin{array}{l}\text { Capacité de stockage } \\
\text { Autres }\end{array}$ & 1 & 4 & 4 & 4 \\
& 5 & 5 & 5 & 5 \\
\hline
\end{tabular}

Figure 29

Utilisateurs des CD-ROM $(n=29)$

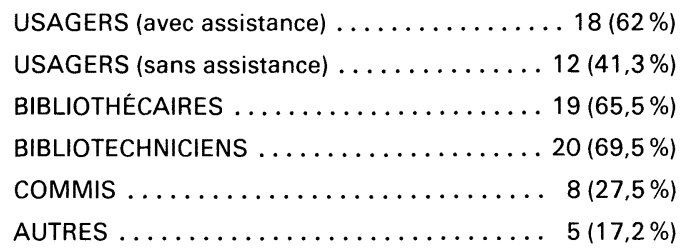

Figure 30

Politique de tarification des services de CD-ROM

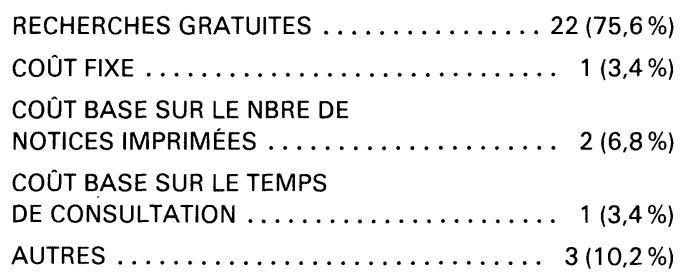


Figure 31

Opinions des répondants (réponses « oui » et «parfois»)

\section{Le CD-ROM :}

est difficile à utiliser par les

usagers eux-mêmes

attire de nouveaux usagers

peut être confondu avec le catalogue

de la bibliothèque

occasionne plus de travail

au personnel

est favorablement accepté

par les usagers
B. publ.

$(\mathbf{n}=\mathbf{3})$

$33,3 \%$

$66,6 \%$

$66,6 \%$

$66,6 \%$

$100 \%$
B. univ.

( $n=11)$

B. spéc.

$(n=15)$

Ensemble

( $n=29$ )

$69,2 \%$

$73,1 \%$

$88,9 \%$

$64,3 \%$

$64,3 \%$

$42,3 \%$

$33,3 \%$

$42,9 \%$

$88,9 \%$

$71,4 \%$

$76,9 \%$

$100 \%$
Figure 32

Problèmes avec le CD-ROM

(réponses " oui » et "parfois»)

\begin{tabular}{|c|c|c|c|c|}
\hline $\begin{array}{l}\text { Type de } \\
\text { problème }\end{array}$ & $\begin{array}{l}\text { B. publ. } \\
(n=2)\end{array}$ & $\begin{array}{l}\text { B. univ. } \\
(n=10)\end{array}$ & $\begin{array}{l}\text { B. spéc. } \\
(n=13)\end{array}$ & $\begin{array}{c}\text { Ensemble } \\
(n=25)\end{array}$ \\
\hline Installation & 0 & $80 \%$ & $69,2 \%$ & $68 \%$ \\
\hline Documentation & 0 & $60 \%$ & $38,5 \%$ & $44 \%$ \\
\hline $\begin{array}{l}\text { Stratégie de } \\
\text { recherche }\end{array}$ & $50 \%$ & $50 \%$ & $76,9 \%$ & $64 \%$ \\
\hline $\begin{array}{l}\text { Termes de } \\
\text { recherche }\end{array}$ & $50 \%$ & $50 \%$ & $46,2 \%$ & $48 \%$ \\
\hline $\begin{array}{l}\text { Logique de } \\
\text { recherche }\end{array}$ & $50 \%$ & $60 \%$ & $61,5 \%$ & $60 \%$ \\
\hline $\begin{array}{l}\text { Visualisation } \\
\text { des résultats }\end{array}$ & 0 & $40 \%$ & $30,8 \%$ & $32 \%$ \\
\hline $\begin{array}{l}\text { Familiarité } \\
\text { avec le } \\
\text { microordinateur }\end{array}$ & 0 & $80 \%$ & $46,2 \%$ & $56 \%$ \\
\hline \multicolumn{5}{|c|}{$\begin{aligned} \text { Autres : - lecteurs de CD-ROM } & \\
& \text { - bases de données différentes en-ligne et sur CD-ROM } \\
& \text { - logiciels d'interrogation faibles } \\
& \text { - besoin d'un disque dur de grande capacité } \\
& \text { - bases de données sur plusieurs disques } \\
& \text { - coût de l'équipement }\end{aligned}$} \\
\hline
\end{tabular}

ROM a eu un impact notable sur leur service de téléréférence (volume de recherche à la baisse) et que plusieurs bibliothèques envisagent d'annuler leur abonnement à la version imprimée des ouvrages acquis sur CD-ROM.

Au Québec, le CD-ROM est encore très peu utilisé dans les bibliothèques. À cet égard, le Québec accuse un retard important sur le Canada et les États-Unis. Un des facteurs prépondérants pour la non-utilisation du CD-ROM semble être l'absence de produits de langue française.
L'apparition, sur CD-ROM, de la base Choix de Services Documentaires Multimedia comblera un peu cette lacune, mais il y a encore énormément de chemin à parcourir pour doter véritablement le Québec d'une CDthèque adaptée. C'est encore malheureusement le phénomène de "l'oeuf et de la poule»: pas de marché, pas de produits, pas de produits, pas de marché. II semble y avoir beaucoup d'attentisme. La plupart des répondants se sont toutefois montrés sinon intéressés, du moins curieux face au CD-ROM. Le taux de réponse au questionnaire $(45,7 \%)$ est d'ailleurs assez élevé pour ce genre d'enquête. En outre, plus de $15 \%$ des répondants qui n'utilisent pas de CD-ROM présentement prévoient en utiliser un dans un proche avenir. Certains projets-pilote sont déjà en cours.

Ce sont les bibliothèques universitaires et les bibliothèques spécialisées qui sont les plus actives dans I'implantation de services de CDROM, un peu comme elles l'avaient été, il y a 10 ou 15 ans, avec les services de téléréférence. D'ailleurs, le parallèle est saisissant avec l'évolution des services de téléréférence. À l'instar de ces derniers, c'est la disponibilité de produits adaptés et pouvant répondre véritablement aux besoins des clientèles qui s'avérera, pour le CD-ROM, la pierre angulaire de leur implantation dans les bibliothèques du Québec. Plusieurs bibliothèques publiques et collégiales, par exemple, ont répondu qu'elles considéreraient faire l'acquisition d'un CD-ROM lorsque les bases de données québécoises seraient disponibles sur ce support. D'ailleurs, pourquoi en irait-il autrement du CD-ROM qu'avec les outils imprimés et les bases de données en-ligne? 


\section{Questionnaire sur les CD-ROM}

S.V.P. cochez toutes les cases appropriées.

1. Type de bibliothèque :
$\square$ municipale
$\square \mathrm{BCP}$
$\square$ universitaire
$\square$ collégiale
$\square$ scolaire
$\square$ gouvernementale
$\square$ spécialisée
$\square$ archives
$\square$ centre de documentation
$\square$ autre (spécifier :

2. Avez-vous un service de téléréférence?

$\square$ oui
$\square$ non

3. Si oui, quels serveurs utilisez-vous présentement ?
$\square$ Dialog
CAN/OLE
$\square$ Wilsonline
$\square$ NLM
$\square$ Orbit
$\square$ IST/Questel
$\square$ BRS
Dow Jones
$\square$ MEAD
$\square$ Infoline
$\square$ Infomart
$\square$ Infoglobe
$\square$ SDM
$\square$ OL Systems
$\square$ UTLAS

$\square$ Autres (spécifier

4. Utilisez-vous présentement une ou des bases de données sur CD-ROM (ex. ERIC, Grolier Encyclopedia, Books in Print, etc.)?

$\square$ oui $\square$ non

Si oui, passez à la question 5 .

Si non, prévoyez-vous en utiliser dans un proche avenir?

$\square$ oui $\quad \square$ non $\square$ ne sais pas

5. Indiquez le nom de vos bases de données sur CD-ROM:

6. Quelles bases de données sur CD-ROM utilisez-vous le plus souvent? (par ordre décroissant):

1.

2.

3.

7. Combien de lecteurs de CD-ROM avez-vous?

8. Indiquez, par ordre de priorité $(1=$ la plus importante, $5=$ la moins importante), les raisons qui vous ont incités à choisir le CB-ROM :

.... facilité d'utilisation des équipements

$\ldots$ interrogation par les usagers eux-mêmes

.... coût fixe des recherches

.... capacité de stockage

.... autre (spécifier : 
9. Selon vos politiques, qui peut utiliser les CD-ROM ?

$\square$ les usagers (avec assistance)

$\square$ les usagers (sans assistance)

$\square$ les bibliothécaires

$\square$ les bibliotechniciens

$\square$ les commis

$\square$ autres (spécifier:

10. Les recherches sur CD-ROM sont-elles:

$\square$ gratuites

$\square$ à coût fixe (combien? .....)

$\square$ à coût basé sur le nombre de notices imprimées

$\square$ à coût basé sur le temps de consultation

$\square$ autre (spécifier :

11. Coyez-vous que le CD-ROM :

oui parfois non ne sais pas

soit difficile à utiliser par les usagers eux-mêmes

attire de nouveaux usagers

puisse être confondu avec le catalogue de la bibliothèque

occasionne plus de travail au personnel

soit favorablement accepté par les usagers

12. Quels sont les principaux problèmes rencontrés avec les CD-ROM?

oui parfois non ne sais pas

installation

documentation

stratégie de recherche

termes de recherche

logique de recherche (boo-

léenne, adjacence, proximité)

visualisation des résultats

familiarité avec le

microordinateur

autres problèmes (spécifier):

13. Commentaires: 